\title{
The Role of NLRP3 in Repairing Sciatic Nerve Injury
}

\author{
Jia Yao ${ }^{1}$, Xiao Gao ${ }^{1}$, Yuzhen Zhu ${ }^{1}$, Xuan Yang ${ }^{1}$, Jianzgao Deng ${ }^{1}$, \\ Bei Zhang ${ }^{1}$
}

${ }^{1}$ Department of Immunology, Medical College of Qingdao University, Qingdao, Shandong 266071, PR China

\begin{abstract}
NLRP3 is a member of the NOD-like family of adaptive immune responses that recognize both exogenous damage-associated molecular patterns and endogenous damage-related molecular patterns. NLRP3 recognizes that PAMPS/DAMPS is activated, binds to ASC and Caspase-1 to form NLRP3 inflammatory vesicles, and exerts immunity in pathogenic infectious diseases and autoimmune diseases by controlling the release of IL-1 $\beta$ and IL-18, but The role of peripheral nerve injury is not clear. The objective of this study is to investigate the effect of NLRP3 on repair after sciatic nerve injury. This study was divided into two parts. The first part established the sciatic nerve injury model. In the second part, NLRP3 intervention was given on the basis of the sciatic nerve injury model. The results showed that The model of sciatic nerve injury in mice was established successfully. The sciatic nerve function of the MSU group and the MCC950 group recovered better than the control group, and the recovery of the MCC950 group was the best. It is concluded that moderate inhibition of nlrp3-mediated inflammatory response is helpful for peripheral nerve repair after injury.
\end{abstract}

Keywords: Nlrp3, Peripheral Nerve Injury, IL-1 $\beta$

\section{Introduction}

Peripheral nerves are mainly composed of somas embedded in the spinal cord, brain stem, dorsal root ganglia, sympathetic ganglia, or parasympathetic ganglia and extended axons that signal with target organs[1]. Pathological changes such as nerve conduction interruption, intra-neuronal edema, and Waller degeneration occur after peripheral nerve injury. Interaction between neurons and immune cells, and their communication and functional cooperation, is integral to homeostasis and species survival[2]. Regeneration after peripheral nerve injury is a complex process influenced by many factors, and the microenvironment of the nerve after injury is an important influencing factor. Innate immunity regulates the microenvironment after peripheral nerve injury by releasing various cytokines and signal transduction effects, thereby affecting regeneration after peripheral nerve injury. It was demonstrated that robust inflammation reactions were acutely induced by peripheral nerve injury[3] , which the inflammatory response can be triggered by a protein complex, an inflammatory corpuscle[4]. The most representative inflammasome is nucleotide binding domain-like receptor protein(NLRP3)[5].

As a key factor in innate immune response,NLRP3 is activated by a variety of endogenous and exogenous stimuli and recruits apoptosis-associated spot-like protein (ASC) to activate caspase1 to stimulate the release of the proinflammatory cytokines interleukin (IL)-1 $\beta$ and IL-18[6]. Interleukin-1 beta is an important class of pro-inflammatory cytokines involved in the inflammatory natural immune response, but its role in the nerve is controversial. Studies have shown that interleukin-1 is not neurotoxic to culture media and normal healthy brain neurons[7]. Some scholars believe that interleukin-1 is the chief culprit of acute neuropathy[8].

In addition to defense against pathogens, NLRP3 inflammatory bodies have been found to be involved in a variety of inflammatory mechanisms, metabolic diseases and peripheral neuropathy. In Alzheimer's disease, IL-1 $\beta$ and IL-18 production can be regulated by regulating NLRP3 degradation, resulting in microglia activation [9], whereas activation of NLRP3 in peripheral nerves can exacerbate neuropathic pain caused by paclitaxel[10]. Other than that, NLRP3 is also closely related to Crohn's disease, diabetes, and heart failure[11-13]. However, whether NLRP3 plays a role in the repair of peripheral nerve injury is not clear, so we can further explore the role of NLRP3 in repairing peripheral nerve injury in the sciatic nerve injury model. The purpose is to elucidate its mechanism of action and provide new ideas for the repair after nerve injury.

This article is published under the terms of the Creative Commons Attribution License 4.0 Author(s) retain the copyright of this article. Publication rights with Alkhaer Publications.

Published at: http://www.ijsciences.com/pub/issue/2019-02/

DOI: 10.18483/ijSci.1925; Online ISSN: 2305-3925; Print ISSN: 2410-4477 


\section{Part I : Construction of a mouse sciatic nerve injury model \\ MATERIALS AND METHODS \\ 1.1 Animals}

All animal experiments were approved by the local Ethics Committee of Qingdao University Medical College. In our study, C57BL/6 mice were purchased from Qingdao Daren Fortune Animal Technology Co. Ltd (Qingdao, China) and either sex was used. The animals were housed 3/cage with free access to food and water in the Qingdao University Boya building's animal room maintained on a 12/12 dark/light cycle at constant temperature $\left(22 \pm 2^{\circ} \mathrm{C}\right)$ and humidity $(60 \%)$

\subsection{Method}

\subsubsection{Peripheral nerve injury}

5-8 weeks old C57BL / 6 mice $(35 \pm 5$ g) were randomly divided into two groups for surgery, including model group and sham operation group. Mice were firstly anesthetized by intraperitoneal injection of $5 \%$ chloral hydrate $(350 \mathrm{mg} / \mathrm{kg})$. The femur was taken as a landmark, the skin and superficial fascia was incised at mid-thigh level and the biceps femoris was separated bluntly to expose the sciatic nerve. The exposed sciatic nerve was clamped with hemostatic forceps at full pressure for $60 \mathrm{~s}$ to transect entire nerve fibers without damaging the epineurium (model group, $n=14$ ). For the sham procedure, the sciatic nerve was only exposed for 60 seconds (s) and remained untouched (sham operation group, $n=14$ ). At last, the skin surgical incision was closed by sutures. the sham operation group only freed the sciatic nerve and then closed the incision. The rats were sacrificed at $4 \mathrm{~h}, 24 \mathrm{~h}$, $48 \mathrm{~h}, 1 \mathrm{~W}, 2 \mathrm{~W}$ and $4 \mathrm{~W}$ after successful modeling. The right sciatic nerves of the two groups of mice were removed at $4 \mathrm{~h}, 24 \mathrm{~h}$ and $48 \mathrm{~h}$ after operation. They were quickly placed in the cryotubes and stored in liquid nitrogen. 1 week, 2 weeks and 4 weeks after the operation, the whole body was perfusion with normal saline for 30 minutes, and then the whole body was perfusion with $40 \mathrm{~g} / \mathrm{L}$ paraformaldehyde. Remove the sciatic nerve. The sciatic nerve was fixed in $40 \mathrm{~g} / \mathrm{L}$ paraformaldehyde for 24 hours and then embedded in paraffin.

\subsubsection{Real-time quantitative PCR for detection of IL-1ßmRNA levels}

the total RNA concentration of reverse transcription was adjusted to $0.05 \mu \mathrm{g} / \mu \mathrm{l}$. Hiscript0r IIQ RT SuperMix for qPCR (+gDNA wiper) (Vazyme, Nanjing, China) were used for cDNA synthesis and qPCR reaction was performed with ChamQ TM Universal SYBR qPCR Master Mix kit (Vazyme, Nanjing, China) according to instructions. The housekeeper gene $\beta$-actin was used as internal reference, and the sequence of primers was shown as follows:
Genes
Forward-primers
Reverse-primers

$\begin{array}{lll}\text { IL-1 } \beta & \text { GCAACTGTTCCTGAACTCAACT } & \text { ATCTTTTGGGGTCCGTCAACT } \\ \beta \text {-actin } & \text { TGTCACCAACTGGGACGATA } & \text { GGGGTGTTGAAGGTCTCAAA }\end{array}$

Reaction conditions: Pre-denaturation at $95{ }^{\circ} \mathrm{C}$ for $10 \mathrm{~s}$, $30 \mathrm{~s}$ at $56{ }^{\circ} \mathrm{C}, 30 \mathrm{~s}$ at $72{ }^{\circ} \mathrm{C}, 40$ cycles. Each

RNA sample was tested for three times, and the average $\mathrm{Cq}$ value was used to analyze the results. The mRNA expression level of IL-1 $\beta$ was quantitatively analyzed by $2-\Delta \Delta$ Cqt method.

\subsubsection{Hematoxylin and eosin (HE) stain}

The paraffin-embedded tissue was cut into thin tissue layers (4- $\mu \mathrm{m}$ thickness) and baked at a high temperature for fixation. The gradient alcohol was held for 3 minutes in accordance with xylene and a series of sequential placements, and then $\mathrm{HE}$ staining was performed according to standard procedures. Finally, DBS reagent was used to reveal the images. The samples of sciatic nerves were observed under a microscope (Nikon, Tokyo, Japan) equipped with a digital camera.

\subsubsection{Behavioral analysis}

The sciatic nerve function index (SFI) was used to estimate the recovery of motor function after sciatic nerve injury. Footprints were analyzed before surgery 1 , 2 , and 4 weeks after surgery. A wooden trough with a length of $60 \mathrm{~cm}$, a width of $10 \mathrm{~cm}$ and a height of 10 $\mathrm{cm}$ was prepared, and $70 \mathrm{~g}$ of white paper was cut into the same width as the wooden trough and then laid on the bottom of the trough. After the pigments of the double hind limbs of the rats were immersed in the ankle joints, the rats were placed at one end of the trough, and they walked to the other side of the trough, leaving 5 to 6 footprints on each hind limb. Selecting the footprints with clear imprints to measure three indicators of normal foot $(\mathrm{N})$ and injured side foot $(\mathrm{E})$ : A, PL (footprint length); B, TS (toe width); C, IT (intermediate toe width). The above index was substituted into the Bain formula to calculate the sciatic nerve function index. Bain formula: SFI $=109.5$ (ETS NTS) / NTS - 38.3 (EPL - NPL) / NPL + 13.3 (EIT NIT) / NIT - 8.8. The SFI measurement ranged from 0 to $\pm 11 \%$ for complete sciatic nerve function, $-100 \%$ for complete loss of sciatic nerve function, and $-11 \%$ to $-100 \%$ for different degrees of sciatic nerve function damage. 


\subsubsection{Statistical analyses}

Statistical analysis was performed using Graph Pad Prism 5.0 software. The experimental data is expressed as mean $\pm \mathrm{SD}$ (standard deviation). One-way ANOVA was used to assess differences between paired data. Bonferroni correction was used when performing independent comparisons. $\mathrm{P}<0.05$ was considered statistically significant according to the criteria of $\alpha=$ 0.05 .

\subsection{Results}

\subsubsection{General condition of mice}

There was no loss in the experimental process, and all of them entered the results analysis. Both groups of rats developed well, no death, invasive grade A healing.

\subsubsection{IL-1 $\beta$ mRNA level in mouse sciatic nerve}

Compared with the sham operation group, IL-1 $\beta$ mRNA expression level was significantly increased in the model group $24 \mathrm{~h}$ after surgery $(\mathrm{P}<0.001)$. This result indicates that IL-1 $\beta$ mRNA expression is up-regulated after sciatic nerve injury. The result is shown in Figure 1.

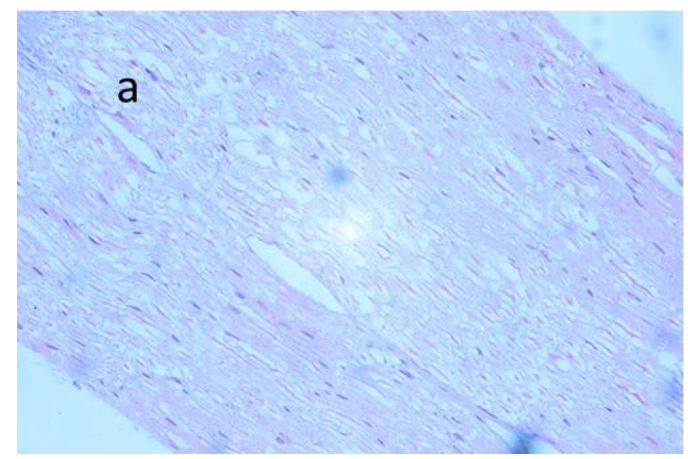

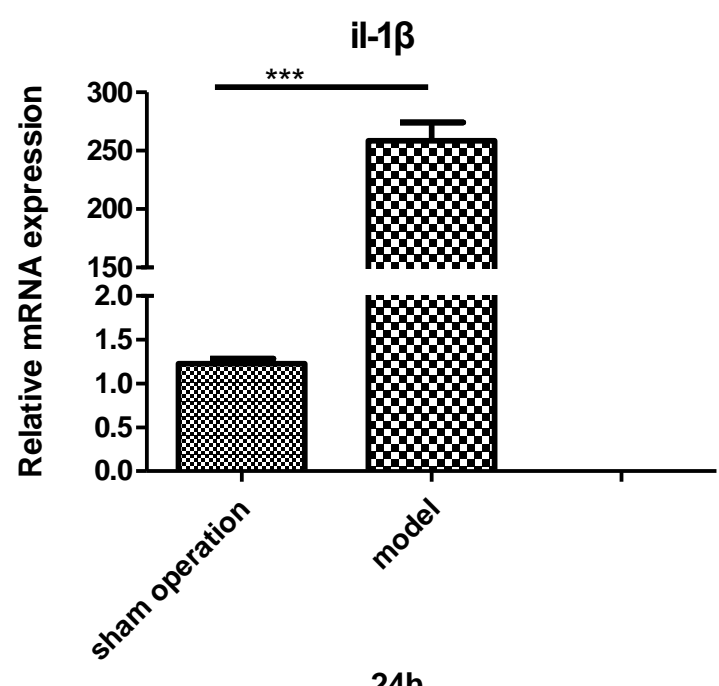

24h

Figure $1 \mathrm{IL}-1 \beta$ mRNA expression level in sciatic nerve tissue of rat.

Note: ${ }^{* * *} \mathrm{P}<0.001$. The difference is significant.

1.3.3 HE staining showed that the model group had obvious nerve damage

HE staining showed that the sciatic nerve tissue of the sham operation group was intact, the fibers were arranged neatly, and there was no inflammatory cell infiltration. The axonal part of the model group was broken, the nerve fibers were disordered, and a large number of inflammatory cells infiltrated. The results are shown in Figure 2.

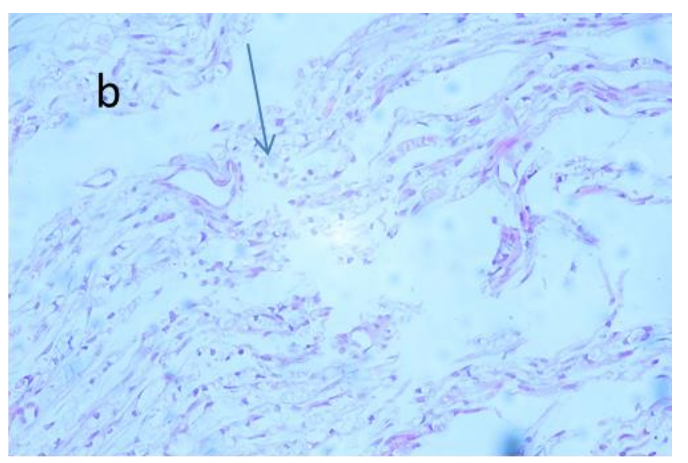

Figure 2 Pathological changes of mouse sciatic nerve tissue (HE staining, $\times 100$ ).

Note: a) sham operation group; b) model group. Arrow pointing to the injury site.

\subsubsection{Sciatic nerve function index}

In the sham operation group and the model group, the sciatic nerve function index was analyzed at 1 week, 2 weeks and 4 weeks after operation, and the recovery of motor function was evaluated. The gait was stable and the footprint was clear when the two groups of mice were walking. The results showed that the sciatic nerve function of the sham operation group was normal. Compared with the sham operation group, the sciatic nerve function index of the model group was significantly lower than that of the sham operation group at 1 week, 2 weeks and 4 weeks after injury. The difference was statistically significant. In the model group, the sciatic nerve function index was the lowest at 1 week after surgery, and then began to increase gradually. This result indicated that the sciatic nerve function of the model group mice was partially lost and gradually recovered. Results are shown in Figure 3. 


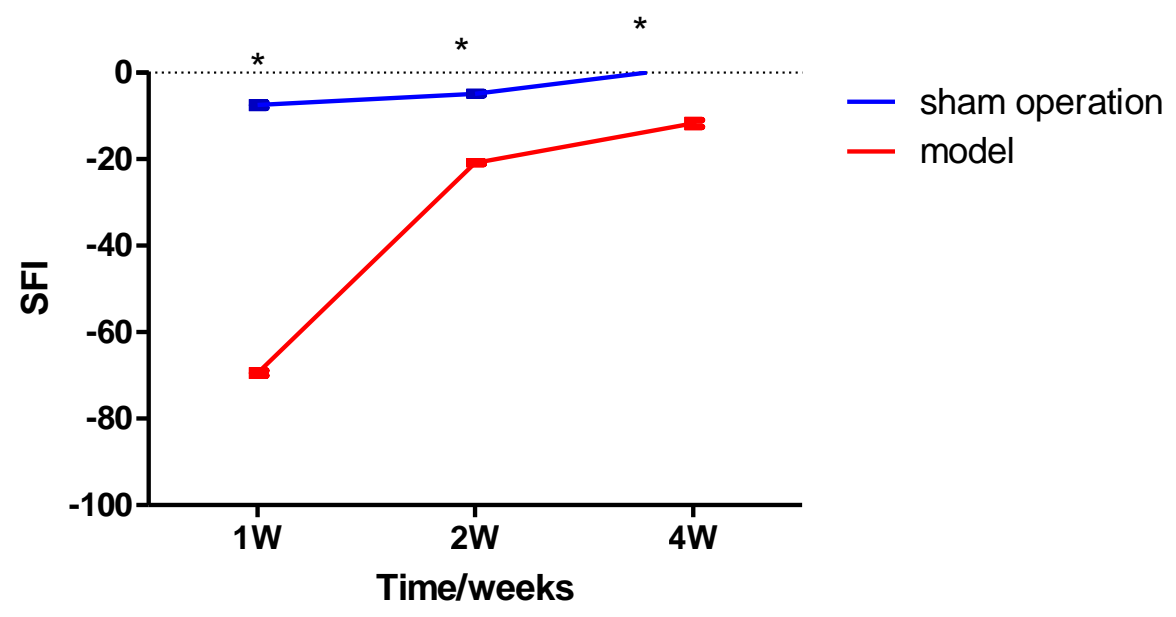

Figure 3 Sciatic nerve function index.

Note: $* \mathrm{P}<0.05$. The difference is significant.

Part II: The role of NLRP3 in the repair of sciatic nerve injury

2.1 Animals

This part is the same as the first part.

\subsection{Method}

\subsubsection{Animal grouping}

Sixty c57BL/6 mice were randomly divided into three groups, 20 in each group, divided into control group (20), MSU group (20) and MCC950 group (20). In the control group, mice in the MSU group and the Mcc950 group were injured by the hemostatic forceps to the right ischial nerve. The MSU group was administered at the injury site $(0.0125 \mathrm{mg} /$ place $)$ half an hour before injury to the sciatic nerve. The MCC950 group was intraperitoneally injected $(10 \mathrm{mg} / \mathrm{kg})$ on days 0,1 and 2 of the sciatic nerve injury and every 2 days thereafter. The control group is injected with a normal amount of physiological saline.

\subsubsection{Sciatic nerve injury model}

This part is the same as the first part.

\subsubsection{Real-time quantitative PCR for detection of IL-1及 mRNA levels}

This part is the same as the first part.

2.2.4 Detection of GAP-43 and P75NTR protein expression levels by immunohistochemistry.

The paraffin-embedded tissue was cut into thin tissue layers $(4-\mu \mathrm{m}$ thickness) and baked at a high temperature for fixation. The gradient alcohol was held for 3 minutes in accordance with xylene and a series of sequential placements, and then immunohistochemistry was assessed to detect the protein level of GAP-43. To this end, the target GAP-43 was repaired by EDTA (1:50). Thereafter, block the tissues with $3 \%$ hydrogen peroxide solution for $10 \mathrm{~min}$ and washed with PBS three times, $5 \mathrm{~min}$ for each. Non-specific antibodies were then sealed with goat serum for 20 minutes at room temperature, excess liquid was removed, incubated with primary antibody for 2 hours at $37{ }^{\circ} \mathrm{C}$, and then washed three times with PBS. After that, incubated tissue section with biotin labeled secondary antibody for $20 \mathrm{~min}$ at RT and washed (3x, 5 min per time) with PBS. Finally, DBS reagent was used to reveal the images. The samples of sciatic nerves were observed under a microscope (Nikon, Tokyo, Japan) equipped with a digital camera.

\subsubsection{Behavioral analysis}

This part is the same as the first part.

\subsubsection{Statistical analyses}

Statistical analysis was performed using Graph Pad Prism 5.0 software. The experimental data is expressed as mean $\pm \mathrm{SD}$ (standard deviation). One-way ANOVA was used to assess differences between paired data. Bonferroni correction was used when performing independent comparisons. $\mathrm{P}<0.05$ was considered statistically significant according to the criteria of $\alpha=$ 0.05 .

\subsection{Results}

2.3.1 IL-1 $\beta$ mRNA and caspase-1 mRNA levels in mouse sciatic nerve

Compared with the control group, the expression level of il-1 mRNA in the mcc950 group was significantly lower at 4 hours after operation, and the difference was statistically significant $(\mathrm{P}<0.001)$. Compared with the control group, the expression of IL-1 $\beta$ mRNA in MCC950 group and MSU group was significantly increased at 24 hours after operation, and reached the highest at 24 hours, and then gradually decreased, the difference was statistically significant $(\mathrm{p}<0.001)$. The above results indicate that MCC950 and MSU drugs successfully interfere with the NLRP3 signaling pathway. Results are shown in Figure 5. 


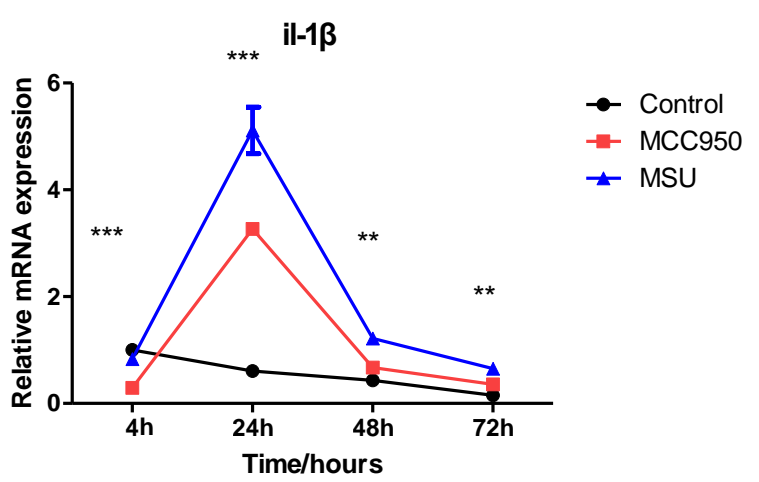

Figure 5 IL-1 $\beta$ mRNA expression levels in sciatic nerve tissue of rat.

Note: $* * * \mathrm{P}<0.001$. $* * \mathrm{p}<0.01$.The difference is significant.

\subsubsection{Expression of GAP-43 proteins in mouse} sciatic nerve

Immunohistochemical detection showed that GAP-43 immuno-positive products, all of which were dark brown, were mainly distributed in the axon, and gap- 43 was mainly located in the cytoplasm, cell membrane and extracellular matrix. By quantitative analysis, the mean value of integral absorbance of immuno-positive cells was calculated. It can be seen that the expression level of MCC950 group and MSU group was increased compared with the control group. Results are shown in Figure 6.

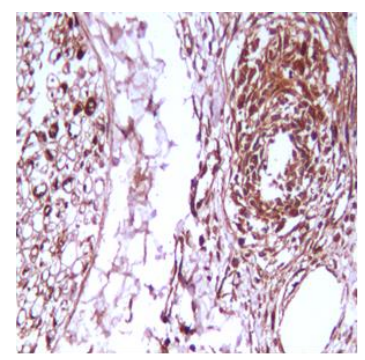

Control

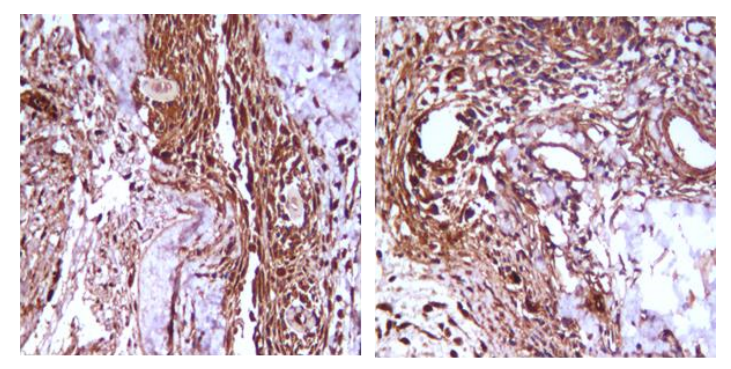

MSU
MCC950

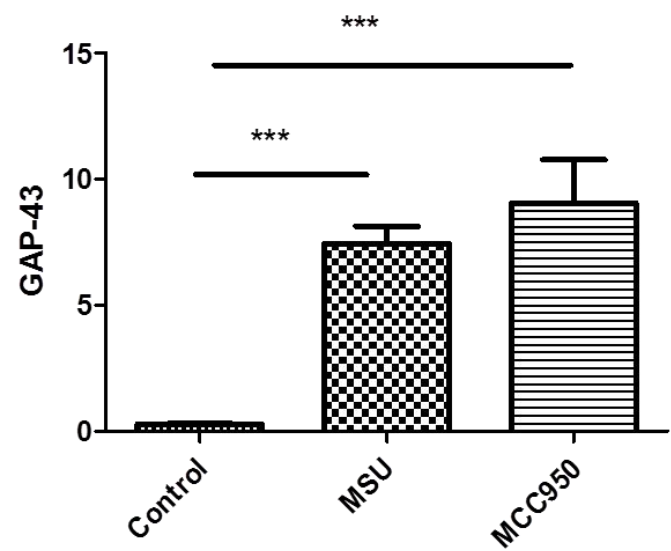

Figure 6 Expression of gap-43 protein in mouse sciatic nerve tissue (immunohistochemistry, $\times 400$ ) Note: $* * * \mathrm{P}<0.001$. The difference is significant.

\subsubsection{Sciatic nerve function index}

The results showed that, compared with the control group, the sciatic nerve function index of the MSU group and the MCC950 group was significantly higher than that of the control group at 1,2 and 4 weeks after injury, and the recovery of the MCC 950 group was better than that of the MSU group. The difference was statistically significant. This result indicated that the recovery of sciatic nerve function in mice expressing NLRP3 was affected. The results are shown in figure 7.

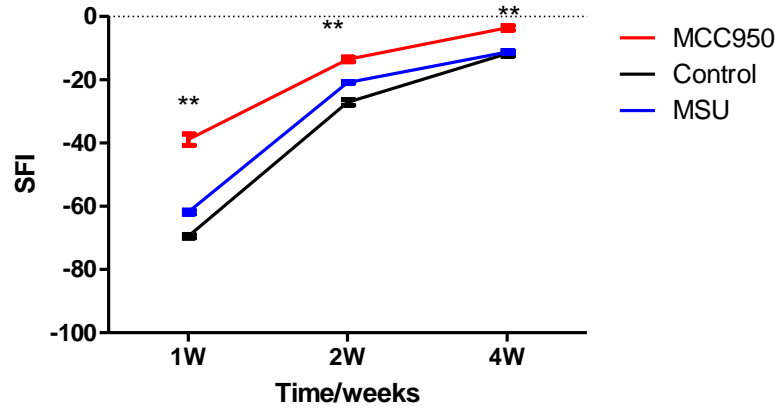


Figure 7 Sciatic nerve function index.

Note: $* * \mathrm{p}<0.01$. $* * * \mathrm{P}<0.001$. The difference is significant.

\section{Discussion}

Many studies have shown that the repair after peripheral nerve injury depends on the microenvironment of the locally damaged nerve[14-16] Several inflammatory cytokines/chemokines were produced 1 hour after peripheral nerve injury, and their expression levels peaked at 24 hours[17]. Therefore, blocking the production and release of inflammatory factors is conducive to the recovery of neural function. Il-1 $\beta$ is a pro-inflammatory cytokine, which was found to increase significantly after sciatic nerve injury in previous studies of our research group, and may be an important cytokine involved in the repair and regeneration of nerve injury. Some studies have claimed that it may cause a large number of il-1ßcentral nervous system ischemia and excitatory intoxication[18], as well as chronic pain and injury of peripheral nerves, where nerve growth is inhibited[19]. However, other studies have shown that the increased

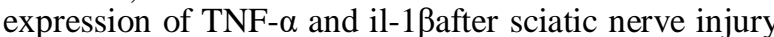
in mice induces neutrophil apoptosis and aggregation, which is beneficial to axonal growth and nerve repair. Conversely, the lack of il-1ßand TNF- $\alpha$ receptors impeded neutrophil recruitment and delayed neurological recovery in mice[20]. Activated NLPR3 can bind to caspase-1 and ASC to form NLRP3 inflammatory corpuscule and induce mature il-1 $\beta$. NLRP3 is closely related to a variety of diseases, such as -amyloid protein in alzheimer's disease, cholesterol crystals in atherosclerosis and so on, but the role of peripheral nerves is not clear.

In this study, NLRP3 was intervened on the basis of the model of sciatic nerve injury in mice, and the relative expression level of il-1ßmRNA was detected. Compared with the control group, the expression level of MSU group was significantly higher at 24 hours, indicating that NLRP3 was activated. However, compared with the control group, the expression level in the MCC950 group was also significantly higher at 24 hours than that in the control group, suggesting that another pathway for the production or release of il-1ßwas compensatory when NLRP3 was inhibited. Development-related protein 43 (gap-43) is an intrinsic determinant of neuronal development and plasticity[21]. The generation of gap-43 is crucial in the reconstruction and extension of axons[22], and plays a role in regulating signal transduction and releasing neurotransmitters.. Immunohistochemistry showed that the expression levels of gap-43 in the NLRP3 intervention group were higher than those in the control group, and the expression levels in the MCC950 group were higher than those in the MSU group. This is negatively correlated with inflammatory infiltration. Therefore, we can speculate that moderate inhibition of NLRP3 can provide a more favorable regeneration microenvironment for regeneration after nerve injury.

\section{References}

1. Romero-Ortega, M., et al., Chronic and low charge injection wireless intraneural stimulation in vivo. Conf Proc IEEE Eng Med Biol Soc, 2015. 2015: p. 1013-6.
2. Chavan, S.S., V.A. Pavlov, and K.J. Tracey, Mechanisms and Therapeutic Relevance of Neuro-immune Communication. Immunity, 2017. 46(6): p. 927-942.

3. Yi, L., et al., Differential gene expression profile of CD4+/CD8+ T cells in patients with hepatitis $\mathrm{C}$ virus and/or human immunodeficiency virus infection. Hong Kong Med J, 2015. 21 Suppl 7: p. S11-3.

4. Zambetti, L.P., et al., The rhapsody of NLRPs: master players of inflammation...and a lot more. Immunol Res, 2012. 53(1-3): p. $78-90$

5. $\mathrm{Xu}, \mathrm{L}$., et al., MiR-34c Ameliorates Neuropathic Pain by Targeting NLRP3 in a Mouse Model of Chronic Constriction Injury. Neuroscience, 2019. 399: p. 125-134.

6. Lamkanfi, M. and V.M. Dixit, Mechanisms and Functions of Inflammasomes. cell 2014. 157(Elsevier Inc. ): p. 1013.

7. Lawrence, C.B., S.M. Allan, and N.J. Rothwell, Interleukin-1 beta and the interleukin-1 receptor antagonist act in the striatum to modify excitotoxic brain damage in the rat. Eur $\mathbf{J}$ Neurosci, 1998. 10(3): p. 1188-95.

8. Brough, D., P.J. Tyrrell, and S.M. Allan, Regulation of interleukin-1 in acute brain injury. Trends Pharmacol Sci, 2011. 32(10): p. 617-22.

9. Houtman, J., et al., Beclin1-driven autophagy modulates the inflammatory response of microglia via NLRP3. EMBO J, 2019.

10. Jia, M., et al., Activation of NLRP3 inflammasome in peripheral nerve contributes to paclitaxel-induced neuropathic pain. Mol Pain, 2017. 13: p. 1744806917719804

11. Mehto, S., et al., The Crohn's Disease Risk Factor IRGM Limits NLRP3 Inflammasome Activation by Impeding Its Assembly and by Mediating Its Selective Autophagy. Mol Cell, 2018.

12. $\mathrm{Xu}, \mathrm{C}$., et al., Targeting of NLRP3 inflammasome with gene editing for the amelioration of inflammatory diseases. Nat Commun, 2018. 9(1): p. 4092

13. Suetomi, T., et al., Inflammation and NLRP3 Inflammasome Activation Initiated in Response to Pressure Overload by $\mathrm{Ca}(2+) / C a l m o d u l i n-D e p e n d e n t$ Protein Kinase II delta Signaling in Cardiomyocytes Are Essential for Adverse Cardiac Remodeling. Circulation, 2018. 138(22): p. 2530-2544.

14. Martini, R. and H. Willison, Neuroinflammation in the peripheral nerve: Cause, modulator, or bystander in peripheral neuropathies? Glia, 2016. 64(4): p. 475-86.

15. Low, P.A., et al., Peripheral nerve microenvironment: collection of endoneurially enriched fluid. Exp Neurol, 1982. 77(1): p. 208-14.

16. Zochodne, D.W., The microenvironment of injured and regenerating peripheral nerves. Muscle Nerve Suppl, 2000. 9: p. S33-8

17. Carroll, S.L.F., Paul W, Expression of JE monocyte chemoattr Source J Neuropathol Exp Neurol SO 1998 Oct $57 \quad 10 \quad 915$ 30[PMIDT9786242]. Journal of Neuropathology and Experimental Neurology, 1998. 57: p. 915-930

18. Hara H, F.R., Gagliardini V, Ayata C, Fink K, Huang Z Shimizu-Sasamata M, Yuan J, Moskowitz MA, Inhibition of interleukin lbeta converting enzyme family proteases reduces ischemic and excitotoxic neuronal damage. National Academy of Sciences, 1997. 94(5): p. 2007-12.

19. Gui, W.S., et al., Interleukin-1beta overproduction is a common cause for neuropathic pain, memory deficit, and depression following peripheral nerve injury in rodents. Mol Pain, 2016. 12.

20. Nadeau, S., et al., Functional recovery after peripheral nerve injury is dependent on the pro-inflammatory cytokines IL-1beta and TNF: implications for neuropathic pain. J Neurosci, 2011. 31(35): p. 12533-42.

21. Routtenberg, B.a.A., GAP 43 an intrinsic determinant of neuronal development and plasticity. Trends Neurosci, 1997. 20(2): p 84-91.

22. Meiri, K.F., K.H. Pfenninger, and M.B. Willard, Growth-associated protein, GAP-43, a polypeptide that is induced when neurons extend axons, is a component of growth cones and corresponds to pp46, a major polypeptide of a subcellular fraction enriched in growth cones. Proc Natl Acad Sci U S A, 1986. 83(10): p. $3537-41$. 